\title{
VALORACIÓN ECONÓMICA DE LA PRODUCCIÓN CIENTÍ FICA EN SALUD EN COLOMBIA. 2000-2005
}

\author{
ECONOMI C VALUATI ON OF SCIENTIFIC PRODUCTI ON I N \\ HEALTH I N COLOMBI A. 2000 - 2005
}

\author{
Nelson Alvis-Guzmán, ${ }^{1 *}$ Ph.D, Fernando De la Hoz, ${ }^{2}$ Ph.D. \\ ${ }^{1}$ Universidad de Cartagena, Facultad de Ciencias Económicas, Departamento de \\ Investigaciones Económicas y Sociales, DIES, Cartagena, Colombia. ${ }^{2}$ Colciencias, \\ Universidad Nacional de Colombia, Departamento de Salud Pública, Bogotá, Colombia. \\ *Correspondencia: nalvis@yahoo.com
}

Recibido: Octubre 16 de 2007; Aceptado: Enero 10 de 2008

\section{RESUMEN}

Objetivo. Valorar económicamente la investigación en salud en Colombia, financiada parcialmente por Colciencias a partir de la gestión del Fondo para la Investigación en Salud (FIS) 2001 - 2005. Materiales y métodos. Se realizó una valoración económica desde la perspectiva de la sociedad y el Estado teniendo en cuenta resultados intermedios, para los que se adoptaron dos escenarios (máximo y mínimo). Resultados. Se revisaron 338 proyectos (48.7\% financiado por Conciencias). El costo medio de los proyectos fue 287 millones. El crecimiento de recursos por proyecto aportados por el FIS fue del $75 \%$ en términos reales y la razón de financiación Colciencias/contrapartida, pasó de 0.8 en 2001 a 1.1 en 2005. Antioquia fue el departamento con mayor participación (30.2\% ). A 2005 habían finalizado 57 ( $16.8 \%$ ) con un costo de 8.889 millones (9.7\%). Como productos se destacan 30 artículos sometidos en revistas nacionales y 35 en internacionales, 69 participaciones en congresos y la formación de 4 doctores y 24 magísteres. La formación de talento humano representa más del $95 \%$ por ciento de los beneficios. La razón beneficio/costo oscila entre 4.72 y 6.34 (por cada peso invertido en investigación en salud se generan, para la sociedad, más de seis veces dicha inversión). Conclusiones. La valoración económica destaca la importancia que en la ejecución de los proyectos de investigación se formen investigadores, frente a los altos retornos que ello implica. Por ello, debe consolidarse, por parte de Colciencias, como criterio de valoración positiva de un proyecto de investigación, el hecho de proponer la formación de investigadores.

Palabras clave: Costo Beneficio, investigación, salud, Colciencias, Colombia. 


\section{ABSTRACT}

Objective. To economically estimate health Research in Colombia, partially financed by Colciencias starting from the administration of the Fund for Research in Health (FRH) 2001 - 2005. Materials and methods. An economic valuation was conducted from perspective of the society and the State, considering intermediate results for which two scenarios were adopted (maximum and minimum). Results. 338 projects were reviewed ( $48.7 \%$ financed by Colciencias). The mean cost per project was 287 millions. The growth of resources for project contributed by the FRH was of $75 \%$ in real terms and the financing ratio Colciencias / counter part, increased from 0.8 in 2001 to 1.1 in 2005. The department with greatest participation was Antioquia (30.2\%), which through 2005 had concluded 57 $(16.8 \%)$ with a cost of 8.889 million $(9.7 \%)$. As products, the following stands out: 30 articles submitted to national journals and 35 submitted to international journals, 69 participations in congresses, and the training of four doctors and 24 Masters of Sciences. The formation of human talent represents more than $95 \%$ percent of the benefits. The benefitcost ratio oscillates between 4.72 and 6.34 (for each monetary unit invested in health research more than six times that investment is generated for society). Conclusions. The economic valuation highlights the important fact that, in the execution of research projects, researchers are trained, in front of the high returns that this implies. For this reason, Colciencias can strengthen the valuation of its funded research projects by considering the positive value of the training of new investigators.

Key words: Benefit-cost, investigation health, Colciencias, Colombia.

\section{NTRODUCI ÓN}

El retorno de la inversión en investigación en salud y la determinación de sus efectos sobre el desarrollo es complejo y poco utilizado. Sin embargo, se han identificado algunas categorías de posibles retornos de la inversión en investigación en salud: i) generación de nuevo conocimiento, ii) beneficios sobre el diseño y evaluación de nueva tecnología, iii) generación de argumentos para diseño de políticas públicas y mejoramiento de la gestión de los sistemas de salud y iv) beneficios económicos más amplios. Por otro lado, se han elaborado varios modelos de uso de la investigación junto con evaluación previa de los retornos o impactos de la investigación en salud y desarrollo (1).

Es evidente que el objetivo básico de la actividad de investigación en salud, como en otras áreas de la ciencia, es la creación de nuevo conocimiento y de nuevos productos y servicios. Tradicionalmente, y en todo el mundo, esta creación de nuevo conocimiento se mide a través de los "productos" (outputs) y de los "efectos" (outcomes). El "producto" (output) de la actividad, adquiere diferentes formas y varía según cada disciplina, desde los artículos en revistas científicas, libros, presentaciones a congresos, y todo tipo de publicaciones en general; patentes y otros productos relacionados con la posibilidad de aplicación y transferencia del conocimiento y, según las áreas, diseños, desarrollo de software, material multimedia, y otros. El producto de la actividad de investigación es generalmente, cuantificable y existen diversas metodologías ampliamente aceptadas, para medirlo. Entre los "resultados" o "efectos" (outcomes) de la investigación se tienen: producción de graduados de alta calidad, innovaciones tecnológicas, ampliación de las capacidades de investigación, relaciones internacionales con potencialidad de intercambio, participación en redes, etc. (2).

Tradicionalmente se usa la bibliometría para medir la producción de los sistemas nacionales de ciencia y tecnología (CTI), a pesar de que ésta, por sí sola, no basta para medir la totalidad de los productos y efectos. Desde hace años, la Red de 
Indicadores de Ciencia y Tecnología I beroamericana e Interamericana- (RICYT), ha compilado datos bibliométricos para ofrecer una visión de la producción científica, en cuanto a publicación de artículos. Si bien éste no es el único de los productos de la actividad de I+D es, sin dudas el más aceptado para medir el producto en ciencia.

\section{Perspectiva económica de la investigación en salud. Desde que el Nóbel de Economía K.J . Arrow en 1963 (3) señaló los principales atributos económicos de la producción de investigación y de la distribución de sus resultados en condiciones de incertidumbre, asimetría de información, opciones alternativas y costos de oportunidad, de alguna manera estuvo determinado el valor económico de la investigación en salud como estrategia para el desarrollo. Así, es cada vez más evidente la importancia del análisis económico para la comprensión de los procesos de asignación de recursos y priorización de la investigación realizada con fondos públicos.}

Sobre la valoración económica de la investigación en salud se puede destacar que: a) se trata de un producto específico y diferenciado por lo que es susceptible de ser valorado económicamente; b) existe necesidad de priorizar la investigación en salud con miras a hacerla consistente con los objetivos del Sistema de Salud y de manera congruente con el perfil epidemiológico, tendiente a generar conocimiento útil y tecnologías aplicables; y c) es necesaria para la rendición pública de cuentas y control democrático de la financiación pública de la investigación en salud, facilitando a los ciudadanos información de forma transparente y suficientemente pormenorizada al objeto de que la consecución de los objetivos estratégicos sea verificable.

El análisis económico es pertinente y aporta información en la comprensión de transacciones entre sujetos con pluralidad (y rivalidad) de intereses y preferencias. A título de ejemplo la definición de rendimiento o impacto multidimensional del proceso de realización y el resultado de la investigación sugerido por Buxton (1) puede ser ilustrativo.
El conocimiento de la complejidad y la multiplicidad de las transacciones entre los diversos agentes en el proceso de investigación en salud permite comprender la importancia de las interrelaciones que presiden el funcionamiento de esta actividad en el seno de las organizaciones sanitarias y desactivar la persistencia de concepciones sesgadas acerca de la "propiedad" de la investigación y en consecuencia modernizar su gestión y concepción estratégica (4).

La gestión de la investigación en salud debe dar tratamiento prioritario al conocimiento permanente de los costos específicos. El conocimiento del valor de la investigación, su costo específico y su costo de oportunidad así como la gestión anticipada del riesgo de elegir dominios tecnológicos y líneas de investigación, constituyen aportaciones distintivas para el análisis económico (5).

El aumento de la conectividad y la reestructuración de la integración vertical de las cadenas de valor en las organizaciones, contribuyen de manera destacada a la visión innovadora de la investigación y de la evolución del ciclo vital del desarrollo del conocimiento científico y su aplicación. Por ciclo vital se entiende el camino realizado o pronosticable de aportación específica de la investigación en salud en el entorno de los servicios sanitarios y en el tiempo, y se define con relación al riesgo y al número de usuarios de las aportaciones científicotécnicas. En la investigación en salud la trayectoria estratégica se refiere al establecimiento de los objetivos y sus productos, y la identidad tecnológica se define a partir de los valores explícitos que mantienen los investigadores con relación a la frontera del conocimiento científicotécnico (6).

La investigación en salud financiada y realizada con fondos públicos posee las características de los bienes públicos en los que el Estado interviene en sustitución del mercado, para producir un servicio de utilización no excluyente y consumo colectivo no rival.

“...el conocimiento presenta dos características notables: su no rivalidad 
y su no exclusividad, una vez que expira el periodo de protección garantizado por el derecho de propiedad intelectual". UNESCO 2005.

Cualesquiera que sean los beneficios que se puedan esperar de la investigación en salud, el conocimiento que ésta genera no se puede asimilar a ningún otro bien intercambiable o negociable en un mercado. En efecto, el conocimiento presenta dos características notables: su no rivalidad y su no exclusividad, una vez que expira el periodo de protección garantizado por el derecho de propiedad intelectual. La primera característica remite a la propiedad del conocimiento, según la cual su utilización por parte de una persona no impide que otra también lo utilice. La segunda característica significa que, a partir del momento en que un conocimiento entra en el dominio público, todos pueden utilizarlo libremente (7).

Se define un "bien público" en el sentido de Samuelson (8) como cualquier ítem que impacta sobre más de una persona, y donde la totalidad del bien impacta a cada persona, ya sean excluibles o no, la investigación en salud, y ante todo el conocimiento que ella genera, es un bien público. El conocimiento propiamente dicho no puede, por lo tanto, ser objeto de una propiedad intelectual exclusiva, y lo que puede entrar en el ámbito del régimen de la propiedad intelectual es la expresión de una idea o una invención, pero nunca las ideas o los hechos originarios que constituyen dicha expresión. En otras palabras, sólo la expresión del conocimiento que adopte la forma de una información puede ser protegida por el derecho de propiedad intelectual, aunque a menudo sea difícil disociar el conocimiento en sí mismo de su expresión formal.

Una vez "producida", sus resultados están a disposición de la sociedad sin exclusiones a un costo de producción prácticamente nulo (aunque su acceso y utilización tengan un costo). Es de especial relevancia que la naturaleza "pública" del "bien” investigación deriva del carácter técnico de su demanda y no de la organización de su producción. A pesar de que la información y el conocimiento aportado por la investigación son apropiables mediante patentes, el sector público parece valorar más la publicación y difusión sin restricciones de los resultados tal vez como mecanismo efectivo de control de calidad vía peer review - y como soporte de la productividad de la futura investigación.

La naturaleza de bien público de la investigación en salud tiene como implicaciones tres cuestiones decisivas para la relevancia social y el control democrático: la explicitación suficiente de los criterios en que se basa la priorización o expresión de la demanda colectiva de investigación, la creación de instrumentos aceptables de valoración de proyectos y de su uso de recursos y el establecimiento transparente del nivel agregado de financiación. Como común denominador de estas dimensiones de apreciación de la eficiencia pública en la asignación de recursos, destaca el dilema acerca del nivel de centralización descentralización de las decisiones de selección y dotación de recursos para proyectos de investigación competitivos.

El objetivo del presente estudio fue de valorar económicamente los resultados de las investigaciones llevadas a cabo con financiación de fondos públicos, con miras a contribuir a la orientación de la gestión de la investigación en salud en Colombia,

\section{MATERI ALES Y MÉTODOS}

Se valoró económicamente la producción de investigación en salud en Colombia entre los años 2001 y 2005. Se tomaron como base de datos los proyectos financiados por Colciencias que fueron presentados en convocatorias abiertas y elegidos con los criterios que para tal fin dispone Colciencias.

Costos de la investigación en salud. Para la valoración de los costos se tomó la perspectiva de la sociedad y el estado, a partir del financiamiento con fondos públicos, de proyectos de investigación. Específicamente en Colombia se han aumentado de manera importante los recursos destinados a la investigación en salud debido a la creación del Fondo de Investigación en Salud - FIS- que se nutre del $7 \%$ de lo producido por las loterías 
departamentales y los juegos de azar (Ley 643 de 2001, Art. 42. Lit. b. reglamentado por el decreto 2878 de 2001).

Para efectos de medición de los costos de investigación se tomaron las asignaciones que Colciencias aprobó para cada proyecto más la respectiva contrapartida de la institución ejecutora. Los costos fueron expresados tanto en pesos corrientes de cada año como en pesos constantes de 2001 que corresponde al año 1 del periodo de análisis.

Beneficios de la investigación en salud. Los beneficios de la investigación en salud pueden discriminarse en distintas categorías de acuerdo a la naturaleza de los resultados. Se pueden definir dos tipos de resultados: Por un lado los que se denominan resultados intermedios referidos a la producción bibliométrica y la formación de talento humano; estos resultados generalmente se producen durante el proceso de investigación. Por otro lado, están los resultados finales que hacen referencia al impacto que el proyecto genera con sus resultados y que generalmente se relacionan con potencialidades del uso del conocimiento, la información y/o la tecnología generada sobre la salud de los individuos o de las poblaciones.

Resultados intermedios. La generación de conocimiento nuevo y contribución a la consolidación y depuración del conocimiento existente es el principal beneficio de la investigación. Dichos beneficios pueden ser medidos a partir de los estudios bibliométricos que muestran la dinámica de las publicaciones (visibilidad del conocimiento). En ello es importante la calidad de la publicación como indicador de validez, por lo que los procesos de indexación han contribuido internacionalmente a fijar estándares sobre el tipo y la calidad del conocimiento generado. Además se toma en cuenta la difusión del conocimiento en la comunidad científico - técnica por otros medios (9). La medición de los resultados intermedios se hace mediante el análisis de las publicaciones en revistas nacionales e internacionales como fruto del trabajo de los grupos de investigación en los distintos proyectos. Además, la participación en eventos o congresos nacionales e internacionales puede ser un indicador de la divulgación del conocimiento entre la comunidad científica.

Otros resultados intermedios tienen que ver con el mejoramiento de los procesos de investigación en cuanto a la capacidad de los grupos para proponer mejores objetivos de investigación, mejorar las habilidades y para desarrollar redes de cooperación y la capacidad para aprovechar el conocimiento existente respecto a la formación de talento humano. Para el presente estudio se midieron estos beneficios a partir de la formación de talento humano en especial estudiantes de doctorados y maestrías.

Resultados finales. Estos tienen que ver con todo lo relacionado con la optimización de los procesos de gestión sanitaria relacionado con: i) reducción de costos en la provisión de servicios, ii) mejoras en la calidad de los servicios, iii) mejoras en la efectividad de las tecnología existentes y la generación de nuevas, iv) mejoras en la equidad tanto en la provisión como en el financiamiento de los servicios de salud, y v) avances en las discusiones éticas y el forjamiento de nuevos paradigmas para el desarrollo de la misma investigación. La medición de estos beneficios implican tanto metodologías como estudios específicos que profundicen en cada uno de dichos beneficios $y$ que merecen ser investigados en otros proyectos.

En tal sentido, para el presente estudio solo se consideró los beneficios en términos de los resultados intermedios que generan los proyectos de investigación. Para efectos de la valoración económica de los resultados se asumieron los siguientes supuestos:

1. Los retornos de una inversión en formación doctoral se distribuyen en la sociedad.

2. Cálculos estimados de COLCIENCIAS muestran que financiar un doctorado en el exterior tienen un costo de US \$120.000 y en el país de US \$50.000 (10). Dado lo anterior la formación de un estudiante de maestría en el exterior tienen un costo de US \$50.000 y en el país de cerca de US $\$ 20.000$. 
3. La información y el conocimiento científico se divulga en medios con mecanismos efectivos de control de calidad - vía peer review - y como soporte de la productividad de la futura investigación. Sin embargo, en materia de valoración económica, los aportes de una publicación en tal sentido, hacen la diferencia de ingresos de los investigadores Así, Colciencias al establecer los parámetros para asignar salarios a los investigadores no solo considera la formación de los mismos sino que el hecho de poseer publicaciones internacionales hace la diferencia de dos salarios mínimos entre magísteres o, entre doctores (11). Así, se asumió como contribución de una publicación la posibilidad que un investigador incremente en dos salarios mínimos su ingreso durante el próximo proyecto. Además, según el decreto 1279 de 2002 (12), por la publicación de un articulo en una revista indexada tipo $A 1$, un investigador recibe hasta 15 puntos (con valor de cerca de $\$ 7.500$ de 2006 , cada punto) con efecto salarial es decir que puede incrementar el salario anual hasta en cerca de un millón setecientos mil pesos (escenario máximo). Dado que el decreto establece condiciones respecto al número de participantes y el tipo de revista, el escenario mínimo es aquel en el que el investigador solo recibe tres puntos, es decir cerca de $\$ 350.000$ anuales.

4. El decreto citado establece que las presentaciones en congresos solo reciben bonificaciones por una sola vez cercanas a los 48 puntos es decir cerca de $\$ 350.000$.

5. Para todos los efectos de las publicaciones se asume un horizonte temporal de 3 años equivalentes a la duración de un proyecto.

6. Para la valoración de los retornos de la formación doctoral y de maestría, se asumió que luego de formados los doctores y/o magísteres se vinculan laboralmente y devengan los salarios establecidos por Colciencias para los investigadores (escenario mínimo para los doctores 11 salarios mínimos mensuales vigentes y para los magísteres 8, y escenario máximo 13,5 y 10 respectivamente).

7. Finalmente, para efectos de la valoración de los retornos de la formación de doctorado y maestría se asumió un horizonte temporal de 20 años.

La información se tomó directamente de las bases de datos del Programa de Ciencia y Tecnología de la Salud de Colciencias y se procesó utilizando una hoja de cálculo de MS Excel $2003{ }^{\circledR}$.

\section{RESULTADOS}

Desde 2001 el Fondo de Investigación en Salud, administrado por Colciencias, ha invertido más de 49 mil millones de pesos en

Tabla 1. Montos de inversión en investigación en salud a través de COLCIENCIAS, Colombia 2001 - 2005 (pesos corrientes).

\begin{tabular}{lcccccc}
\hline \multicolumn{1}{c}{ AÑO } & $\mathbf{2 0 0 1}$ & $\mathbf{2 0 0 2}$ & $\mathbf{2 0 0 3}$ & $\mathbf{2 0 0 4}$ & $\mathbf{2 0 0 5}$ & Total \\
\hline Proyectos presentados & 292 & 310 & 265 & 292 & 295 & 1454 \\
Proyectos financiados & 77 & 93 & 21 & 113 & 34 & 338 \\
Proporción & 26.4 & 30.0 & 7.9 & 38.7 & 11.5 & 23.2 \\
\hline COLđENCIAS - FIS & 7.579 .555 & 9.935 .394 & 3.485 .051 & 21.000 .000 & 7.284 .730 & 49.284 .730 \\
Promedio & 98.436 & 106.832 & 165.955 & 185.841 & 214.257 & 145.813 \\
Desv. Estándar & 42.102 & 38.196 & 103.190 & 106.422 & 105.617 & 91.306 \\
\hline Contrapartida & 9.525 .990 & 11.897 .069 & 5.230 .814 & 18.575 .677 & 6.749 .953 & 51.979 .503 \\
Promedio & 123.714 & 129.316 & 249.086 & 165.854 & 198.528 & 154.701 \\
Desv. Estándar & 109.494 & 150.514 & 319.035 & 128.602 & 89.630 & 151.085 \\
\hline Monto Total & 17.105 .544 & 21.832 .463 & 8.715 .866 & 39.575 .677 & 14.034 .683 & 101.264 .233 \\
Promedio & 222.150 & 234.758 & 415.041 & 350.227 & 412.785 & 299.598 \\
Desv. Estándar & 133.400 & 166.902 & 387.616 & 212.450 & 178.116 & 211.734 \\
\hline
\end{tabular}


la financiación de 338 proyectos de investigación científica y desarrollo tecnológico en diferentes aspectos de la salud humana en los departamentos, municipios y distritos de Colombia (Tabla1).

El crecimiento del promedio de los recursos por proyecto aportados por el FIS ha sido del $75 \%$ en términos reales y la razón de financiación Colciencias/contrapartida, pasó de 0.8 en 2001 a 1.1 en 2005 (Figura 1), esto se refleja en el incremento del promedio de duración de los proyectos durante el período (Tabla 2).

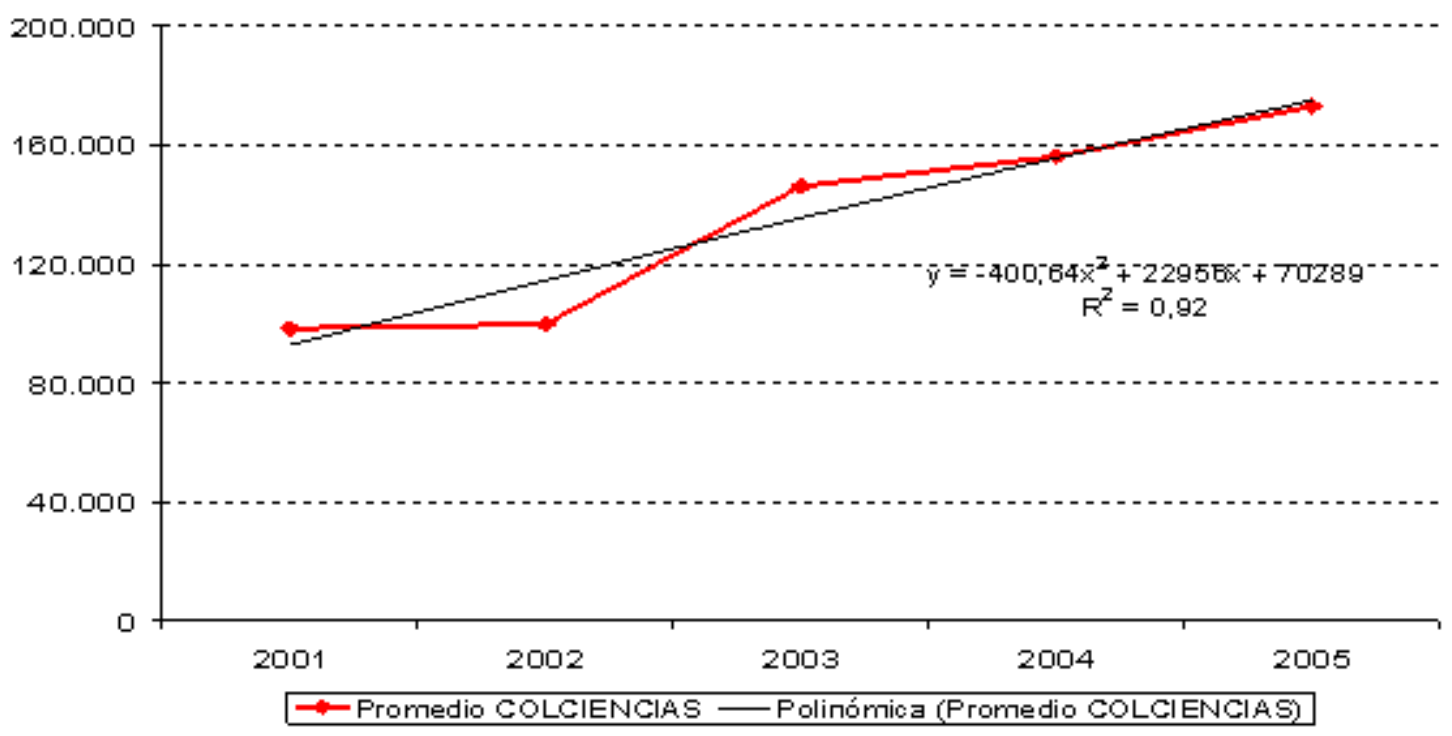

Figura 1. Crecimiento de la financiación de los proyectos de investigación en salud gestionados a través de COLCIENCIAS, Colombia 2001 - 2005 (pesos constantes de 2001).

Tabla 2. Montos de inversión en investigación en salud a través de COLCIENCIAS, Colombia 2001 - 2005 (pesos constantes de 2001).

\begin{tabular}{lcccccc}
\hline \multicolumn{1}{c}{ AÑO } & $\mathbf{2 0 0 1}$ & $\mathbf{2 0 0 2}$ & $\mathbf{2 0 0 3}$ & $\mathbf{2 0 0 4}$ & $\mathbf{2 0 0 5}$ & Total \\
\hline Proyedos & 77 & 93 & 21 & 113 & 34 & 338 \\
\hline COLCIENCIAS - FIS & 7.579 .555 & 9.286 .283 & 3.071 .071 & 17.650 .025 & 5.882 .847 & 43.469 .781 \\
Promedio & 98.436 & 99.853 & 146.241 & 156.195 & 173.025 & 673.750 \\
Desv. Estándar & 42.102 & 35.701 & 90.933 & 89.445 & 85.292 & 343.473 \\
\hline Contrapartida & 9.525 .990 & 11.119 .795 & 4.609 .459 & 15.612 .437 & 5.450 .984 & 46.318 .665 \\
Promedio & 123.714 & 120.867 & 219.498 & 139.397 & 160.323 & 763.799 \\
Desv. Estándar & 109.494 & 140.681 & 281.138 & 108.087 & 72.382 & 711.781 \\
\hline Monto Total & 17.105 .544 & 20.406 .078 & 7.680 .530 & 33.262 .462 & 11.333 .831 & 89.788 .445 \\
Promedio & 222.150 & 219.420 & 365.740 & 294.358 & 333.348 & 1.435 .016 \\
Desv. Estándar & 133.400 & 155.997 & 341.572 & 178.559 & 143.839 & 953.368 \\
\hline
\end{tabular}

El promedio anual de duración de los proyectos se pasó de 28.1 meses en el 2001 a 31.6 el en 2005, es decir, solo un incremento del $12 \%$ lo cual contrasta con el incremento de los costos medios por proyecto que fue del $75 \%$ en términos reales. Esto implica una probable mayor complejidad de dichos proyectos relacionados con el uso de tecnologías y la intensificación del talento humano participante. Es decir, cada vez los proyectos son ejecutados por grupos en el que participan más de 5 investigadores y más de una institución (Tabla 3).

Por otro lado, la participación de los departamentos pasó de 8 en el 2001 a 13 
Tabla 3. Duración media según fecha de inicio de los proyectos de investigación en salud financiados a través de COLCIENCIAS, Colombia 2001 - 2005.

\begin{tabular}{ccc}
\hline Año & Duración media de los proyectos (meses) & IC 95\% \\
\hline 2001 & 28.1 & $26.5-29.8$ \\
2002 & 26.4 & $24.8-28.0$ \\
2003 & 27.2 & $23.0-31.3$ \\
2004 & 30.0 & $28.3-31.6$ \\
2005 & 31.6 & $29.2-34.0$ \\
\hline Promedio general & 28.6 & $27.7-29.5$ \\
\hline
\end{tabular}

en el 2005, manteniéndose Antioquia como el departamento con mayor participación en la gestión de proyectos de investigación en salud (30.2\%), le siguen en importancia el Distrito Capital y el departamento del Valle (Tabla 4).
En cuanto a las instituciones que ejecutan posproyectos, la Universidad de Antioquia continúa siendo líder con una participación cerca de tres veces mayor que sus seguidoras, la Universidad Industrial de Santander, el Instituto Nacional de Salud y

Tabla 4. Proyectos de investigación en salud financiados a través de COLCIENCIAS, Colombia 2001 - 2005.

\begin{tabular}{|c|c|c|c|c|c|c|c|}
\hline DPTO & 2001 & 2002 & 2003 & 2004 & 2005 & Total & $\%$ \\
\hline 1. Antioquia & 26 & 23 & 6 & 37 & 10 & 102 & 30.2 \\
\hline 2. Bogota d.c. & 24 & 28 & 7 & 32 & 7 & 98 & 29.0 \\
\hline 3. Valle & 11 & 15 & 3 & 24 & 1 & 54 & 16.0 \\
\hline 4. Santander & 12 & 21 & 3 & 9 & 7 & 52 & 15.4 \\
\hline 5. Bolívar & & & & 6 & & 6 & 1.8 \\
\hline 6. Caldas & 1 & 1 & & 1 & 2 & 5 & 1.5 \\
\hline 7. Cauca & 1 & 2 & & 1 & 1 & 5 & 1.5 \\
\hline 8. Risaralda & 1 & & & 2 & 1 & 4 & 1.2 \\
\hline 9. Atlántico & & 1 & 1 & 1 & & 3 & 0.9 \\
\hline 10. Quindío & & 1 & 1 & & 1 & 3 & 0.9 \\
\hline 11. Tolima & 1 & 1 & & & & 2 & 0.6 \\
\hline 12. Boyacá & & & & & 1 & 1 & 0.3 \\
\hline 13. Córdoba & & & & & 1 & 1 & 0.3 \\
\hline 14. Nariño & & & & & 1 & 1 & 0.3 \\
\hline 15. Norte de Santander & & & & & 1 & 1 & 0.3 \\
\hline Total general & 77 & 93 & 21 & 113 & 34 & 338 & 100 \\
\hline
\end{tabular}

la Universidad del Valle. Es importante observar que la participación de la Universidad del Valle se concentró en el año 2004 , mientras que el resto de instituciones tiene una participación anual más regular. Llama la atención el descenso en la participación del Instituto Nacional de Salud el cual paso de ejecutar 9 proyectos en el 2001 a 1 en le 2005. Por otro lado, la universidad nacional de Colombia y el CIDEIM se mantienen constantes en su participación anual (Tabla 5).
Con relación al enfoque de los proyectos ejecutados ha predominado el biomédico, aunque su tendencia ha sido decreciente en el período, gracias a la participación cada vez más importante de los enfoques clínico y epidemiológico. Las investigaciones en sistemas y políticas de salud han marcado una tendencia creciente y se posicionan con una participación importante en el 2005 (17.6\%) (Figura 2). 
Tabla 5. Institución que ejecutaron proyectos de investigación en salud financiados a través de COLCIENCIAS, Colombia 2001 - 2005.

\begin{tabular}{|c|c|c|c|c|c|c|c|}
\hline Entidad & 2001 & 2002 & 2003 & 2004 & 2005 & Total & $\%$ \\
\hline Universidad de Antioquia & 19 & 18 & 5 & 28 & 7 & 77 & 22.8 \\
\hline Universidad Industrial de Santander - UIS & 4 & 15 & 3 & 4 & 3 & 29 & 8.6 \\
\hline Instituto Nacional de Salud & 9 & 7 & 1 & 2 & 1 & 20 & 5.9 \\
\hline Universidad del Valle - UNIVAUE & & & & 16 & & 16 & 4.7 \\
\hline वDEM & 4 & 5 & 2 & 4 & & 15 & 4.4 \\
\hline Fundación Cerdiovascular De Colombia & 5 & 4 & & 2 & 4 & 15 & 4.4 \\
\hline Universidad del Valle & 7 & 7 & & & & 14 & 4.1 \\
\hline Universidad Nacional de Colombia & 2 & 2 & 2 & 5 & 2 & 13 & 3.8 \\
\hline Corporación para Investigaciones Biológicas - $\mathrm{CE}$ & 5 & 3 & & 4 & & 12 & 3.6 \\
\hline Universidad de los Andes & 4 & 4 & & 3 & 1 & 12 & 3,6 \\
\hline Pontificia Universidad Javeriana & 2 & 5 & & 2 & & 9 & 2.7 \\
\hline Universidad Autónoma de Eucaramanga & 3 & 1 & & 3 & & 7 & 2.1 \\
\hline FUNDA,CÓN OARDIO INFANTIL - F.CI & 1 & 2 & & 3 & & 6 & 1.8 \\
\hline FUNDA,CON PARA, LA, EDUCAGON SUPERIIOR. & 1 & 1 & & 4 & & 6 & 1.8 \\
\hline FUNDAGON SANTA FE DE BOGOTA - CES & & & & 6 & & 6 & 1.8 \\
\hline Instituto Nacional de Cencerología & 2 & 3 & 1 & & & 6 & 1.8 \\
\hline Universidad El Bosque & 1 & 2 & & 2 & 1 & 6 & 1.8 \\
\hline Universidad del Cauca & 1 & 2 & & 1 & 1 & 5 & 1.5 \\
\hline Universidad Tecnológica de Pereira & 1 & & & 2 & 1 & 4 & 1.2 \\
\hline Instituto de Ciencias de la Salud - $C$ E & 1 & & & 2 & & 3 & 0.9 \\
\hline Universidad - Colegio Mayor de Nuestra Señora del Rosario & & & 1 & 2 & & 3 & 0.9 \\
\hline Universidad De Cartagena & & & & 3 & & 3 & 0.9 \\
\hline Universidad del Norte & & 1 & 1 & 1 & & 3 & 0.9 \\
\hline Universidad del Quindio & & 1 & 1 & & 1 & 3 & 0.9 \\
\hline Corporación Universitaria Del Sinú & & & & 2 & & 2 & 0.6 \\
\hline Instituto Colombiano de Mediana Tropical - CES & & & & & 2 & 2 & 0.6 \\
\hline Instituto Nacional de Cancerología & & & & 2 & & 2 & 0.6 \\
\hline Universidad de Caldas & & 1 & & & 1 & 2 & 0.6 \\
\hline Universidad de Caldas & 1 & & & 1 & & 2 & 0.6 \\
\hline Universidad del Tolima & 1 & 1 & & & & 2 & 0.6 \\
\hline Acaldía de Duitama-Secretaria de Salud & & & & & 1 & 1 & 0.3 \\
\hline Asociación Colombiana dela Salud - ASSALUD & & & 1 & & & 1 & 0.3 \\
\hline Asociación de Alergia, Asma e Inmunología & 1 & & & & & 1 & 0.3 \\
\hline Centro intemadonal de educacion y desarrollo humano & & & & & 1 & 1 & 0.3 \\
\hline Centro intema cional de física - $\square F$ & & 1 & & & & 1 & 0.3 \\
\hline Centro intemadonal de vadunas - MVDC & & & 1 & & & 1 & 0.3 \\
\hline Cínica Reina Sofia & & & & 1 & & 1 & 0.3 \\
\hline Corporación CORPOGEN & & & & & 1 & 1 & 0.3 \\
\hline CORPORA,GÓN UNIVERSTAARIA.R.AFAEL NÚÑEZ - Grtagena & & & & 1 & & 1 & 0.3 \\
\hline FEDESALUD, Bogotá & & & & 1 & & 1 & 0.3 \\
\hline Fundación Canguro & & 1 & & & & 1 & 0.3 \\
\hline $\begin{array}{l}\text { Fundación grupo latinoamericano para la participación, la integración } \\
\text { y la inclusión de las personas con discapaddad - GLARP.IIPD }\end{array}$ & & & & 1 & & 1 & 0.3 \\
\hline Fundación Hospitalaria San Vicente de Paúl & & & & & 1 & 1 & 0.3 \\
\hline Fundación Neumológica Colombiana & & & 1 & & & 1 & 0.3 \\
\hline Fundación Santa Fe de Bogotá & 1 & & & & & 1 & 0.3 \\
\hline Fundación Universitaria de Genda y Tecnologia "Konrad Lorenz" & & 1 & & & & 1 & 0.3 \\
\hline FUNDAGON VAULE DEL LILI & & 1 & & & & 1 & 0.3 \\
\hline
\end{tabular}


HOSPTAL PABLO TCEON URIBE

Instituto Colombiano de Mediana Tropical "Antonio Roldán

Betancour"

Instituto Colombiano De Medicina Tropical "Antonio Roldan

Betancour - - ICMT Sabaneta, Antioquía.

Instituto colombiano de medicina tropical "Antonio Roldan

Betancour ${ }^{-}$- ICMT, Medellín

Instituto Nacional de Cancerologia. Bogotá.

Instituto para Niños Gegos y Sordos del Valle del Cauca

Laboratorio de Investigación Clínica Molecular - LIOM Cali

Universidad de Córdoba

Universidad de Nariño

Universidad de Pamplona

Universidad de San Buenaventura

Universidad Esauela de Administradón y Finanzas y Tecnológicas.

EAFIT

Universidad Libre de Cali

Universidad Nacional de Colombia-Sede Madellín

Universidad Pedagógica y Tecnológica de Colombia

Universidad pontificia bolivariana sede Bucaramanga

Total general

1

1

\begin{tabular}{ccccc} 
& & & 1 & 0.3 \\
& & & 1 & 0.3 \\
& 1 & & 1 & 0.3 \\
& 1 & & 1 & 0.3 \\
& 1 & & 1 & 0.3 \\
& & 1 & 1 & 0.3 \\
& 1 & & 1 & 0.3 \\
& & 1 & 1 & 0.3 \\
& & 1 & 1 & 0.3 \\
& & 1 & 1 & 0.3 \\
1 & & & 1 & 0.3 \\
& & & 1 & 0.3 \\
& & & 1 & 0.3 \\
& 1 & & 1 & 0.3 \\
& & 1 & 1 & 0.3 \\
& & & 1 & 0.3 \\
\hline $\mathbf{2 1}$ & $\mathbf{1 1 3}$ & $\mathbf{3 4}$ & $\mathbf{3 3 8}$ & $\mathbf{1 0 0}$ \\
\hline & & & &
\end{tabular}

Figura 2. Enfoque de los proyectos de investigación en salud gestionados a través de COLCIENCIAS, Colombia 2001 - 2005.

Los costos medios de los proyectos varían según el enfoque, siendo los más altos los de sistemas de salud y los más bajos los de enfoque de salud pública. De igual modo, su duración varia con el enfoque aunque las diferencias no sean representativas (Tabla 6).

Respecto a los beneficios generados por los proyectos terminados se cuenta que a
31 de diciembre de 2005 han finalizado 57 (16.8\%) de los 338 aprobados en el período. Estos representan un costo de 8.889 millones de pesos corrientes $(9.7 \%)$.

Como productos de estos 57 proyectos, se destacan 30 artículos sometidos en revistas nacionales y 35 en internacionales lo cual muestra una razón de un poco mas de un artículo por proyecto. Se cuentan 69 
Tabla 6. Duración media de los proyectos de investigación en salud financiados a través de COLCIENCIAS, Colombia 2001 - 2005.

\begin{tabular}{lccc}
\hline \multicolumn{1}{c}{ Enfoque } & $\begin{array}{c}\text { Duración media de los } \\
\text { proyectos (meses) }\end{array}$ & IC 95\% & $\begin{array}{c}\text { Costo medio por } \\
\text { proyecto }\end{array}$ \\
\hline Básico Biomédico & 30.5 & $29.2-31.8$ & $292.293 .288,60$ \\
Clínico & 29.8 & $28.0-31.7$ & $309.028 .783,40$ \\
Epidemiológico & 26.5 & $24.5-28.5$ & $321.211 .192,60$ \\
Salud Pública & 23.6 & $21.2-26.0$ & $171.846 .212,10$ \\
Sistemas de Salud & 26.2 & $23.5-29.0$ & $350.954 .694,10$ \\
\hline Promedio general & 28.6 & $27.7-29.5$ & $299.598 .322,50$ \\
\hline
\end{tabular}

participaciones en congresos y la formación de 4 doctores y 24 magísteres (Tabla 7).

\section{Valoración económica de los beneficios ( resultados intermedios). Asumiendo los supuestos explicitados en la metodología, se presentan dos escenarios (mínimo y máximo) de la valoración económica de los resultados intermedios que se resumen en la (Tabla 8).}

Se observa como para los dos escenarios, los aportes mas importantes estuvieron dados por la formación de talento humano el cual representa, en términos económicos, cerca del $95 \%$ de los beneficios. Esto confirma la importancia de los escenarios de ejecución de proyectos de investigación como elementos de formación del talento humano en salud.

Respecto a la razón beneficio/costo esta oscila entre 4.72 y 6.34 lo cual significa que por cada peso que se invierte en investigación en salud se estaría generando para la sociedad, un poco más de seis veces dicha inversión.

Sin embargo, cuando se asume que todos los proyectos de investigación implican la formación de investigadores a nivel de maestrías o doctorados, la razón beneficio/ costo puede oscilar entre 15.7 y 20.6 lo cual implica un incremento entre tres y cuatro veces los beneficios, respecto a las condiciones actuales.

Tabla 7. Productos de los proyectos de investigación en salud finalizados que fueron financiados a través de COLCIENCIAS, Colombia 2001 - 2005.

\begin{tabular}{lcccc}
\hline \multicolumn{1}{c}{ AN̉O DE INICIO } & $\mathbf{2 0 0 1}$ & $\mathbf{2 0 0 2}$ & $\mathbf{2 0 0 3}$ & Total \\
\hline Proyectos & 3 & 33 & 21 & 57 \\
Articulo en revista nacional & & 21 & 9 & 30 \\
Articulo en revista internacional & 2 & 19 & 14 & 35 \\
Participación en Congresos Nacionales & 2 & 22 & 15 & 39 \\
Congresos en Congresos Internacionales & 2 & 19 & 9 & 30 \\
Formación de estudiantes de Doctorados & & 4 & 4 \\
Formación de estudiantes de Maestría & & 13 & 11 & 24 \\
Formación de estudiantes de Pregrado & 1 & 7 & 8 & 16 \\
Formación de estudiantes de Postgrado & & 6 & 2 & 8 \\
Formación de Jóvenes Investigadores & & 2 & 3 & 5 \\
Financiación CoLCIENCIAS (en miles de pesos corrientes) & 90.000 & 2.523 .483 & 1.713 .9724 .327 .456 \\
Contrapartida (en miles de pesos corrientes) & 514.4582 .647 .058 & 1.400 .5834 .562 .099 \\
Monto total de financiación (en miles de pesos corrientes) & 604.4585 .170 .5423 .114 .5568 .889 .555 \\
\hline
\end{tabular}


Tabla 8. Valoración económica de los resultados intermedios de los proyectos de investigación en salud financiados a través de COLCIENCIAS, Colombia 2001 - 2003.

\begin{tabular}{|c|c|c|c|c|c|}
\hline Escenario Máximo & 2001 & 2002 & 2003 & Total & $\%$ \\
\hline Articulo Nacional & - & 109.856 .250 & 47.081 .250 & 156.937 .500 & 0.4 \\
\hline Articulo internacional & 10.462 .500 & 99.393 .750 & 73.237 .500 & 183.093 .750 & 0.4 \\
\hline Participación en Congresos $\mathrm{Nal}$. & 700.000 & 7.700 .000 & 5.250 .000 & 13.650 .000 & 0 \\
\hline Participación en Congresos Interne & 700.000 & 6.650 .000 & 3.150 .000 & 10.500 .000 & 0 \\
\hline Doctorado & - & 490.000 .000 & - & 490.000 .000 & 1.2 \\
\hline Maestría & - & 637.000 .000 & 539.000 .000 & 1.176 .000 .000 & 2.8 \\
\hline Retornos del Doctorado & - & 6.576 .960 .000 & - & 6.576 .960 .000 & 15.7 \\
\hline Ret ornos de la maest ría & - & 18.086 .640 .000 & 15.304 .080 .000 & 33.390 .720 .000 & 79.5 \\
\hline TOTAL BENEFIGOS & 11.862 .500 & 26.014 .200 .000 & 15.971 .798 .750 & 41.997 .861 .250 & 100 \\
\hline RAZON BENEFICIOS/COSTOS & 0.02 & 5.03 & 5.13 & 4.72 & \\
\hline \multicolumn{6}{|l|}{ Escenario Mínimo } \\
\hline Articulo Nacional & - & 21.971 .250 & 9.416 .250 & 31.387 .500 & 0.1 \\
\hline Articulo internacional & 2.092 .500 & 19.878 .750 & 14.647 .500 & 36.618 .750 & 0.1 \\
\hline Participación en Congresos Nal. & 700.000 & 7.700 .000 & 5.250 .000 & 13.650 .000 & 0 \\
\hline Participación en Congresos Interne & 700.000 & 6.650 .000 & 3.150 .000 & 10.500 .000 & 0 \\
\hline Doctorado & - & 490.000 .000 & - & 490.000 .000 & 0.9 \\
\hline Maestía & - & 637.000 .000 & 539.000 .000 & 1.176 .000 .000 & 2.1 \\
\hline Retornos del Doctorado & - & 16.442 .400 .000 & 13.912 .800 .000 & 30.355 .200 .000 & 53.8 \\
\hline Retornos de la maest ría & - & 13.153 .920 .000 & 11.130 .240 .000 & 24.284 .160 .000 & 43.1 \\
\hline TOTAL BENEFICOS & 3.492 .500 & 30.779 .520 .000 & 25.614 .503 .750 & 56.397 .516 .250 & 100 \\
\hline RAZON BENEFICIOS/COSTOS & 0.01 & 5.95 & 8.22 & 6.34 & \\
\hline
\end{tabular}

\section{DISCUSIÓN}

La modelización del rendimiento de la investigación se ha basado en un esquema de análisis input - output, para formalizar las fases de mayor significado estratégico en la cadena de valor de la investigación y su proceso de producción y aplicación.

La naturaleza de bien público de la investigación en salud sustituye a la preferencia individual como criterio de asignación de recursos, cobrando valor central la valoración de la calidad y la relevancia científico - técnica de las aportaciones de la investigación y la evaluación "inter pares" basada en la relación de agencia que se establece entre la sociedad y la comunidad científica, que mantienen posiciones asimétricas en cuanto a la posesión de información relevante y la actuación difícilmente verificable del investigador.
Sin embargo, la anterior noción de necesidad - basada enteramente en los valores, criterios e intereses de los propios investigadores - ha abierto paso a un nuevo concepto más equilibrado. Sus criterios de asignación de recursos consideran explícita y simultáneamente las siguientes dimensiones: una descripción suficiente de la existencia de oportunidades de avance en el conocimiento o en la aportación de evidencia relevante y novedosa; la constatación verificable de la existencia de potencial para la contribución positiva de los recursos existentes - humanos y tecnológicos - a la consecución de los objetivos establecidos y el costo de oportunidad de las alternativas de investigación relevantes, así como la explicitación del criterio de dilucidación entre las mismas (6). 
En los últimos años ha habido un desarrollo teórico significativo y algunas experiencias muy innovadoras para evaluar el retorno (payback) de la investigación. Los modelos desarrollados intentan medir no solamente la contribución específica en el cuerpo de conocimientos de la ciencia (resultados primarios), sino también avances en el proceso de investigación, beneficios para las instituciones dedicadas a la actividad científica e impactos políticos, administrativos, sanitarios y económicos (1). Dado que los beneficios de la investigación pueden adoptar varias formas de retorno o impacto, su valoración requiere un constructo multidimensional y de diferentes metodologías (13).

Por ultimo, con base en este estudio se puede concluir:

1. Es evidente la factibilidad de valorar económicamente los resultados de las investigaciones en salud, en especial lo relacionado con los productos intermedios.

2. Son evidentes las limitaciones de esta metodología para valorar la totalidad de los resultados, en especial en el mediano y largo plazo, por lo que se hace necesario de análisis complementarios que contribuyan a reforzar los hallazgos.

3. Este tipo de valoración permite visualizar la importancia que en la ejecución de los proyectos de investigación se formen estudiantes de doctorado y/o maestrías, frente a los altos retornos que ello implica. Por ello, debe consolidarse, por parte de Colciencias, como criterio de valoración positiva de un proyecto de investigación, el hecho de proponer la formación de investigadores.

4. El análisis de los impactos de largo plazo de los resultados de investigación está reservado para metodologías mas amplias y específicas para cada caso.

5. Sin embargo, la valoración económica es una herramienta que aporta, en el corto plazo, los insumos necesarios para tomar decisiones de inversión en investigación y desarrollo.

\section{REFERENCIAS}

1 Buxton, M., and Hanney, S. How can payback from health services research be assessed. J Health Serv Res Policy 1996; (1): 35-43.

2 Anna María Prat. La importancia de medir la producción científica. [fecha de acceso Enero 12 de 2007]. URL disponible: http://www.ricyt.org/ interior/difusion/pubs/elc2003/8.pdf 2007.

3 Arrow Kenneth J. Uncertainty and the Welfare Economics of Medical Care. Am Econ Rev 1963; (53): 941-973.

4 Fundación para la Investigación y la Docencia de los Hospitales del Valle de Hebrón. Recomendaciones del Plan Estratégico (1999-2004) de I nvestigación Biomédica, en Fundación
Salud, I. y. S., ed. Seminario: La Modernización de la Estrategia y la Gestión de la Investigación Biomédica Madrid: 2000.

5 Culyer Tony. Economics and Public Policy: Research and Development as a Public Good. Reforming Markets in Health Care: An Economic Perspective. Buckingham: Smith Peter C, Open University Press: 2000.

6 Artells J. Estrategia y asignación de recursos en la investigación biomédica. Gac Sanit 2000; (14): 391-397.

7 Baker $\mathrm{M}$, and Kirk S. Research and Development for the NHS: evidence, evaluation and effectiveness. Oxford: Radcliffe Medical Press 1999. 
8 Samuelson Paul A. The Pure Theory of Public Expenditure. Rev Econ Stat 1954; (36): 387-389.

9 Grant J, Cottrell R, Cluzeau F, and G., F. Evaluating "payback" on biomedical research from papers cited in clinical guidelines: applied bibliometric study," Br Med J 2000; (320): 1107-1111.

10 DNP. CONPES 3179: Política Integral de Apoyo a los Programas de Doctorados Nacionales. Bogotá D.C: Departamento Nacional de Planeación, 15 de julio de 2002 .
11 COLCIENCIAS. Guía para la presentación de proyectos de investigación científica y tecnológica. Modalidad de financiación: Recuperación contingente. Bogotá D.C: Colciencias, Abril 22 de 2005.

12 Decreto 1279 de Junio 19 de 2002. Bogotá D.C. Colombia: Ministerio de Educación Nacional; 2002.

13 Camí J. Evaluación de la investigación biomédica, Med Clin 2001; 117: 510513. 